\title{
Revisión sistemática del uso de agentes físicos de pacientes pediátricos basado en la evidencia científica
}

\section{Systematic review of the use of physical agents in pediatric patients based on scientific evidence}

\author{
JAIME-ESTRADA, Sandra $\dagger^{*} \&$ SASIA-ZAYAS, Karen \\ Universidad Tecnológica del Suroeste de Guanajuato (UTSOE) \\ ID 1 ${ }^{\text {er }}$ Autor: Sandra, Jaime-Estrada / ORC ID: 0000-0003-4208-8306 \\ ID $1^{\text {er }}$ Coautor: Karen, Sasia-Zayas / ORC ID: 0000-0001-7243-0753
}

DOI: $10.35429 / J N T .2020 .12 .4 .1 .13$

Recibido 03 de Octubre, 2020; Aceptado 30 de Diciembre, 2020

\section{Resumen}

Los agentes físicos son una forma de energía que tienen la capacidad de interactuar con la materia, y se utilizan en el proceso de rehabilitación, entre ellos podemos encontrar el uso de agua, calor, frío, sonido, corrientes eléctricas, y radiación eletromagnética. El objetivo principal de este estudio es analizar si es benéfico el uso de los agentes físicos en la intervención e interacción con los pacientes pediátricos (0 a 17 años. Material y métodos: Se realizó una extensa búsqueda de material bibliográfico en las bases de datos Google Académico, Medigraphic, Physiotherapy Evidence Database (PEDro), Pubmed, se realizó entre el 06 de julio y el 30 de octubre del 2020, con los límites: estudios en pacientes pediátricos, escritos en idioma español e inglés, que fueran de tipo: revisiones sistemáticas, estudios controlados y aleatorios, y guías de práctica. Resultados: De los 1860 estudios analizados, solamente 45 se pudieron agregar para esta investigación ya que cumplían con los criterios de inclusión. Se pudo comprobar la hipótesis inicial: los agentes físicos son eficaces en el tratamiento de los pacientes pediátricos, sin embargo se considera que falta más investigación en este rubro.

Pediátricos, Agentes físicos, Fisioterapia

\begin{abstract}
Physical agents are a form of energy that have the ability to interfere with matter, and are used in the process of rehabilitation, among them we can find the use of water, heat, cold, sound, electrical currents, and electromagnetic radiation. The main objective of this study is to analyze if is beneficial the use of physical agents in the intervention and interaction with pediatric patients (0 to 17 years). Material and methods: An extensive search of bibliographic material in Google Academic, Medigraphic, Physiotherapy Evidence Database (PEDro), Pubmed, was conducted between July 6 and October 30, 2020, with the limits: studies in pediatric patients, written in Spanish and English, which were of the type: systematic reviews, controlled and randomized studies, and practice guidelines. Results: Of the 1860 studies analyzed, only 45 could be added for this research since they met the inclusion criteria. The initial hypothesis could be tested: physical agents are effective in the treatment of pediatric patients, however it is considered that more research is needed in this area.
\end{abstract}

Pediatrics, Physical agents, Physiotherapy

Citación: JAIME-ESTRADA, Sandra \& SASIA-ZAYAS, Karen. Revisión sistemática del uso de agentes físicos de pacientes pediátricos basado en la evidencia científica. Revista de Técnicas de la Enfermería y Salud. 2020, 4-12: 1-13

$\dagger$ Investigador contribuyendo como primer autor. 


\section{Introducción}

Los agentes físicos son formas de energía que tienen la capacidad de interactuar con la materia, influyendo de manera directa en las respuestas fisiológicas y orgánicas del cuerpo humano, por tal motivo se utilizan en la intervención fisioterapéutica, con la finalidad de mejorar los procesos patológicos o secuelas predisponentes en los pacientes.

Entre los principales agentes físicos utilizados en el proceso de rehabilitación podemos encontrar el uso de agua, calor, frío, sonido, corrientes eléctricas y radiación electromagnética. Y aunque en la actualidad, algunos autores consideran que los agentes físicos son puramente empíricos o un simple placebo, el aumento de publicaciones científicas ha llevado a un incremento en el conocimiento de la efectividad de estos (Ulloa 2009), conllevando a la importancia e implementación de su utilización como complemento terapéutico.

El objetivo principal de este estudio sistemático de revisión bibliográfica, surge de la necesidad de analizar si es benéfico o no el uso de los agentes físicos en la intervención e interacción fisioterapéutica con los pacientes pediátricos (0 a 17 años) sin generar efectos adversos sobre el organismo y desarrollo del paciente.

\section{Agentes físicos en pacientes pediátricos: para entender la importancia de su uso, se deben conocer los efectos que ocasionan respuestas en el organismo del paciente, para facilitar su aplicación y restricciones de los mismos:}

1.1 Efectos biológicos: vasoconstricción o vasodilatación, aumento de extensibilidad en los tejidos, disminución de rigidez articular, analgesia, disminución de espasmos musculares, disminución de la inflamación, aumento/disminución del metabolismo (Camerón 2015).
1.2 Efectos metabólicos: aumento y estimulación de la síntesis de ATP intracelular y el ADN, aumento de la síntesis proteica y enzimática, normalización del potencial de acción, aumento de nutrientes y oxígeno, incremento de elementos defensivos humorales y celulares, aumento del trofismo y mejora de los procesos de reparación (Ulloa 2009).

Para facilitar su revisión se dividen en los siguientes subtemas:

\section{Uso del ultrasonido terapéutico en pacientes pediátricos:}

El ultrasonido terapéutico se define como la terapia que usa energía ultrasónica para producir cambios en los tejidos mediante efectos mecánicos o térmicos (Almirón 2019), los mismos que dependerán según su frecuencia, potencia y ciclo de trabajo en las diversas aplicaciones, buscando favorecer a la disminución de las secuelas de se pudieran presentar de la patología.

\section{Ultrasonido terapéutico en niños con parálisis cerebral tipo diparesia espástica y espástica leve:}

La parálisis cerebral se puede definir como un grupo de trastornos del desarrollo del movimiento y la postura, causantes de la limitación de la actividad, atribuido principalmente a una agresión en la época fetal o durante los primeros años de vida, sobre el cerebro en desarrollo (Poó 2008). De las secuelas que esta patología preceden, se pueden encontrar los siguientes resultados que confirman la efectividad de este agente.

El ultrasonido terapéutico de baja intensidad ( $3 \mathrm{~Hz}$ ) más 3 ciclos de ejercicio de estiramiento pasivo mantenido en la musculatura tratada, durante 6 meses (divididos en dos bloques de 10 sesiones) con un periodo de descanso de 15 días en 11 pacientes, son un complemento eficaz para el tratamiento de las contracturas y acortamientos musculares, favoreciendo así al aumento en el rango óptimo de movimiento en la articulación de la rodilla (Arriaga 2010). 
Reafirmando la efectividad del uso del ultrasonido en esta patología y los beneficios que de ella proceden, Cigarroa (2016) en su población con 12 pacientes pediátricos de entre 7 y 12 años de edad, con parálisis cerebral tipo espástica leve con acortamiento de la musculatura isquiotibial. Con el protocolo de tratamiento de un grupo que recibió movilización de triple flexión, masoterapia y ultrasonido terapéutico de baja intensidad $(3 \mathrm{~Hz})$ y finalizando con una serie de 3 estiramientos pasivos estáticos y en cambio el otro grupo que recibió la misma intervención excluyendo el ultrasonido, con una frecuencia de 2 veces por semana durante 4 semanas, aunado a todos los beneficios ya mencionados se evitó también deformidades articulares en pacientes con parálisis cerebral.

Por lo tanto se puede considerar como una técnica complementaria útil para la disminución de los trastornos del aparato musculoesquelético en los pacientes con este tipo de desorden.

\section{Uso de técnicas especializadas de hidroterapia en pacientes pediátricos:}

La hidroterapia se define como la utilización del agua como agente terapéutico para producir en el organismo efectos térmicos, mecánicos, fisiológicos y biológicos. Aguirre en el 2014 refiere que la terapia acuática es una forma de terapia física que puede ser empleada para tratar a niños y adolescentes con discapacidad, ya que gracias a sus características únicas ofrece posibilidades que son difíciles de alcanzar en tierra.

Estas respuestas se obtendrán dependiendo de la técnica, método y tiempo de aplicación, para esta revisión bibliográfica se utilizaron los siguientes métodos:

\subsection{Técnica Hallwick en pacientes pediátricos con parálisis cerebral infantil:}

Como ya se había abordado anteriormente, la parálisis cerebral es un grupo de trastornos del desarrollo del movimiento y la postura, causantes de la limitación de la actividad (Poó 2008).
Guevara en el 2013 realiza la implementación hidroterapéutica con la técnica Halliwick, la cual está desarrollada, mediante 10 pasos al cumplimiento del objetivo de enseñar a pacientes con discapacidad (física) a nadar y a ser independientes en el agua, aumentando su participación e independencia. Aportando grandes beneficios en cuanto al estado físico, mental y psicológico, de los 30 pacientes atendidos con esta modalidad, durante un periodo de 6 meses, 2 veces por semana, por un periodo de 45 minutos, de los cuales se repartió en 5 minutos para el cambio de ropa, 15 en un ajuste mental, 20 minutos en ejercicios terapéuticos y tareas dirigidas, y 5 minutos para el aseo y cambio, generado los siguientes resultados:

- $100 \%$ obtuvieron una excelente adaptación y ajuste mental al medio acuático, mejoraron su estado cardiovascular y de retorno venoso, se favoreció la relajación muscular (mejorando la movilidad articular), normalización del tono, inhibición de los patrones anormales, facilitando la movilidad y por ende el control postural estático y dinámico.

- $\quad 70 \%$ obtuvieron un control respiratorio en el medio acuático y terrestre.

- $53 \%$ obtuvieron un aumento en la participación, integración social y autoestima, brindando una mejor calidad de vida en los niños de 0-17 años.

Así mismo, Pérez en el 2018 en su participación aporta que: este método es más específico y seguro que las técnicas en tierra, que es beneficioso para mejorar la función motora gruesa, control postural, equilibrio funcional, coordinación, potencia, precisión, flexibilidad, fuerza muscular en miembros superiores, disminución de la espasticidad y aumento en la condición cardiorrespiratoria.

Comprobando así la eficacia de la aplicación del Concepto Halliwick en los pacientes con este tipo de trastorno, no solo en el ámbito físico sino también repercutiendo directamente sobre el estado emocional y afectivo del paciente. 


\subsection{Técnica de Bad Ragaz implementada en pacientes pediátricos con Síndrome de Down y parálisis cerebral infantil:}

El síndrome de Down (SD) es un trastorno genético causado por la presencia de una copia extra del cromosoma 21 (o una parte del mismo), en lugar de dos habitualmente (trisomía del par 21), caracterizado por la presencia de un grado variable de retraso mental y rasgos físicos peculiares que le dan un aspecto reconocible (Basile 2008).

La aplicación de la técnica Bad Ragaz, la cual, utiliza las propiedades del agua para crear un programa para la ejecución de patrones de resistencia, fortalecimiento, reeducación muscular, alargamiento del tronco, relajación e inhibición del tono, propiocepción y analgesia. Mediante patrones en espiral diagonal.

Demostrado que en el total de los 20 pacientes con un rango de edad de los 8 a los 9 años, de los cuales $55 \%$ presentan hipotonía (63.63\% hombres y $36.36 \%$ de mujeres) los resultados de que se mejora en el $90,9 \%$ de los pacientes sometidos a este tratamiento el tono muscular, beneficiando el desarrollo motor normal y permitiendo un equilibrio muscular funcional para mejorar su calidad de vida y desarrollo de su capacidad funcional normal (Chicaiza 2016).

Apoyando los beneficios de esta técnica, Andrade (2016) realizó un estudio a 35 niños y niñas con parálisis cerebral espástica en un rango de edad de 1 a 5 años, argumentando y concluyendo que es una excelente alternativa de tratamiento para el paciente neurológico ya que los resultados se pueden observar en menor tiempo que un tratamiento convencional, obteniendo la disminución del tono muscular, mejorando la movilidad y flexibilidad de la musculatura, incrementar los arcos de movilidad en las extremidades y el fortaleciendo y reeducación de los grupos musculares, mejorando con ello la coordinación y equilibrio de la marcha fuera del mismo.

Concluyendo así con que la finalidad de esta técnica hidroterapéutica es ganar el mayor grado de independencia del paciente pediátrico dentro del medio acuático y facilitando la ejecución de los ejercicios, teniendo mejores resultados para la integración de nuevos patrones motores que fuera del medio acuático.

\subsection{Método Watsu en niños con parálisis cerebral infantil:}

Esta técnica es, según el estudio de Chicaiza (2014) muy efectivo para disminuir el tono, por ello el paciente pediátrico con esta patología, donde usualmente se presentan espasmos, tiende a aumentar la flexibilidad de la musculatura afectada al aplicarse esta técnica, para obtener las siguientes implicaciones:
- En el paciente pediátrico con PC hipotónico, el esta técnica probablemente no le beneficiará, ya que tiene a la disminución en el tono muscular.
- En el paciente pediátrico con PC atetosica los beneficios incluyen la relajación, el aumento de la movilidad del tejido blando y normalización del tono muscular.
- $\quad$ En el paciente pediátrico con PC atáxica se observó mejoría en la relajación y movilidad del tejido blando.

Concluyendo que dentro de los beneficios de la implementación de la terapia acuática, independientemente de la patología predisponente que pueda presentar el paciente, se encuentran: mejora y potenciación de la función pulmonar y cardiovascular, aumento y desarrollo de la función motora gruesa, regulación del tono muscular, aumento y mantenimiento del rango óptimo de movimiento, aumento de la calidad de vida y estado funcional de independencia.

\section{Electroterapia en pacientes pediátricos}

La electroterapia, por definición, consiste en la aplicación de energía electromagnética al organismo (de diferentes formas), con el fin de producir sobre él reacciones biológicas y fisiológicas, las cuales serán aprovechadas para mejorar los distintos tejidos cuando se encuentran sometidos a enfermedad $\mathrm{O}$ alteraciones metabólicas de las células que componen dichos tejidos, que a su vez forman el organismo vivo humano y animal en general. (Meneses, 2010). 
Al momento de la aplicación de electroterapia en pacientes pediátricos se tienen que tener ciertas consideraciones tanto del usuario, como de la corriente, respecto al paciente, se deben tener en cuenta factores: morfológicos (que la zona de aplicación en el pediátrico sea ideal), fisiológicos (estado de la piel, procesos óseos, heridas, exceso de grasa, etc.), psicológicos (que al infante no le de miedo la aplicación, o le genere una fobia), así como la sensibilidad (León, 2005, p. 66)

\section{Estimulación eléctrica funcional (EEF) en pacientes pediátricos según la evidencia}

La denominación de estimulación eléctrica funcional (EFF) se aplica cuando la corriente eléctrica logra contraer al músculo con la fuerza necesaria y en el momento adecuado para que este pueda cumplir alguna función (agarrar y soltar, pararse, caminar, etc.). A diferencia de la estimulación eléctrica terapéutica (ETT) en la cual se busca mejorar alguna condición intrínseca de los músculos y articulaciones (fuerza, espasticidad, contracturas) (Sotelano, 2003, p.137).

Irene Moll et al (2017), realiza una revisión sistemática utilizando la metodología de la Academia Americana de Parálisis Cerebral y Medicina del Desarrollo y las pautas de los Elementos de Información Preferidos para Revisiones Sistemáticas y Meta-Análisis (PRISMA) para evaluar el efecto de la EFF en dorsiflexores del tobillo en niños y adolescentes con parálisis cerebral espástica durante la marcha, obteniendo como resultados, que de 780 resúmenes solamente 5 artículos fueron de nivel I a III de evidencia, donde hay pruebas limitadas de una disminución en la frecuencia reportada de arrastres y caídas de los dedos de los pies.

Los resultados se clasificaron según la Clasificación Internacional del Funcionamiento, la Discapacidad y la Salud (CIF). En el nivel de estructura y función corporal de la CIF, hay pruebas claras (I-III) de que el EFF aumentó el ángulo de dorsiflexión del tobillo (activo), la fuerza y mejoró el control motor selectivo, el equilibrio y la cinemática de la marcha. Algo que se puede resaltar del estudio de Irene Moll es que los efectos de la EFF apuntan hacia un papel potencial como alternativa a las órtesis para los pacientes con parálisis cerebral espástica (Moll et al, 2017)
La EEF es una intervención muy conocida que se utiliza durante la marcha para mejorar la capacidad de caminar y corregir las desviaciones de la marcha facilitando el grupo muscular adecuado en el momento oportuno del ciclo de marcha, Khamis en el 2017, evalúan 15 estudios que cumplieron con los criterios de inclusión. La EFF estimuló los músculos flexores de la dorsal con un efecto ortopédico positivo, mejoró la flexión de la dorsal durante la fase de impulsión y mejoró el patrón de contacto del pie. Se encontró un efecto positivo más pequeño para la estimulación de los extensores de rodilla que facilitaba la extensión de la rodilla durante la fase de apoyo y para la estimulación de los abductores de la cadera que mejoraba la alineación de la rodilla en el plano frontal. No se encontraron pruebas que apoyaran el uso de la estimulación de los flexores plantares para corregir las desviaciones de la marcha (Khamis, et al 2017).

Para la disfunción intestinal vejiga en niños Anne Wrigth en el 2016, utilizando FES. Como resultados obtuvieron que se necesitan más ensayos aleatorios de alta calidad y resultados a largo plazo para establecer si la electroestimulación es capaz de alterar la trayectoria a largo plazo de un niño con disfunción vesical e intestinal (Wrigth et al, 2016)

En un estudio publicado en el 2015, la estimulación nerviosa tibial percutánea y la estimulación nerviosa sacral (SNS) combinado con toxina botulínica, dan resultados favorables, explicando que no se requiere una respuesta motora, si no que basta con una respuesta sensorial (Tubaro, et al 2015).

\section{Estimulación Eléctrica Recíproca (RES)}

Elnaggar et al. (2019) realiza un estudio donde analiza los efectos independientes frente a los integrados de la RES y el botulismo (BoNT). Realiza un ensayo aleatorio a ciegas donde con una población de 60 niños con diplejía espástica se les asignaron intervenciones de RES, BoNT, o de RES Y BoNT integradas (20 niños por cada grupo) 
Todos los niños participaron en un programa de ejercicio de 60 minutos 3 veces por semana, durante 12 semanas sucesivas. Además, el grupo RES recibió estimulación eléctrica recíproca en los flexores de tobillos durante 30 minutos antes de cada sesión de ejercicio, el grupo BoNT fue inyectado por toxina botulínica A en los músculos de la pantorrilla una semana antes de comenzar el programa de ejercicios y el grupo integrado de RES y BoNT recibió ambas intervenciones.

La cinemática de la articulación del tobillo (ángulo de desplazamiento en el contacto inicial, máxima posición de dorsiflexión y pico de oscilación de dorsiflexión) y los límites dinámicos de la estabilidad postural (anterior/posterior [APLOS], medial/lateral [ML-LOS] y general [OLOS]) se evaluaron en entrada y después de la intervención.

Los resultados en este estudio fueron que mediante medidas estadísticas se concluye que la integración de RES y BoNT tiene la capacidad de restaurar la estabilidad postural y la cinemática del tobillo en niños con diplejía. Lo observado en este grupo y como impacto en la rehabilitación clínica es que tiene un efecto considerable en algunos de los elementos esenciales que contribuyen a la mejora de la biomecánica del tobillo y la estabilidad postural. El efecto demostrado proporciona la base para su aplicación en el tratamiento de la diplejía espástica.

\section{Estimulación Eléctrica Transcutánea. (TENS)}

Se realizó un estudio de intervención no aleatorio con 26 niños con vejiga neurogénica debido a la enfermedad de mielomeningocele, de entre 5 y 15 años de edad. A cada niño se le realizó un estudio urodinámico. A cada niño se le realizo un UDS de rutina, y luego un segundo UDS, durante el cual se les aplicó la estimulación eléctrica en la región parasacral. El resultado principal fue la diferencia en la presión máxima de la vejiga observada entre los dos estudios urodinámicos, analizados a partir de la prueba de la t emparejada.
Una de las conclusiones más significativas con el uso de la estimulación eléctrica transcutánea es que tuvo un efecto inmediato significativo en la reducción de la presión máxima de la vejiga durante los estudios urodinámicos entre los pacientes pediátricos con vejiga neurogénica probados. Los resultados fueron más significativos entre los niños menores de 12 años de edad (Dombek et al., 2019)

En el caso del estreñimiento funcional existe un estudio presentado por Rebeca Mayara Padilha Rego en el 2019 a través de la estimulación eléctrica transcutánea del nervio tibial posterior. Los pacientes fueron sometidos a una estimulación nerviosa eléctrica transcutánea del nervio tibial posterior, basada en el método descrito por Queralto y cols en 2006. Esta intervención fue supervisada por un fisioterapeuta. Se aplica con un electrodo autoadhesivo (electrodo positivo) aproximadamente de 3 a $4 \mathrm{~cm}$ por encima del maléolo medial de la tibia, y se colocará un segundo electrodo (negativo) justo debajo del maléolo medial de la misma pierna. Los electrodos se conectaron a un dispositivo de estimulación eléctrica (canales de TENS/FES, Neurodyn Portable Ibramed).

La posición del electrodo se determinará mediante la visualización de la flexión rítmica de los dedos de los pies durante la estimulación. El nivel de intensidad se determina en función de la intensidad inferior al umbral de máxima sensibilidad del paciente, normalmente entre 10 y 25 Ma. Se aplicó simultáneamente una corriente de 200-us, $30 \mathrm{~Hz}$, en los dos miembros inferiores, 30 minutos diarios durante 4 semanas consecutivas. Los resultados demuestran la eficacia del método para aumentar el número de evacuaciones intestinales y la consistencia de las heces, reducir el número de episodios de incontinencia fecal retentiva, e indirectamente mejorar la calidad de vida en general (Padilha, 2019).

\section{Corriente Interferencial}

Al buscar evidencia de la corriente interferencial (CI), se encontró un artículo de Zivkovic en 2017, en el cual se evalúan los efectos de la CI y los ejercicios de respiración diafragmática en niños con disfunción de la vejiga y el intestino. 
Se incluyeron en el estudio clínico prospectivo 79 niños con vaciamiento disfuncional y estreñimiento crónico que fueron fracasos de las intervenciones de atención primario. A todos los niños se les revisó su historial médico con respecto a los síntomas del tracto urinario inferior y los hábitos intestinales. Los niños llevaron un diario de la vejiga y el intestino, y se sometieron a análisis y cultivos de orina, a un examen de ultrasonido de la vejiga y los riñones, y a una uroflujometría con electromiografía del suelo pélvico.

Los niños elegibles se dividieron en 3 grupos (A, B y C). A todos los grupos se les asignó educación y modificaciones de comportamiento. Además, el grupo A fue sometido a ejercicios diafragmático y estimulación con CI, mientras que el grupo B sólo recibió ejercicio de respiración diafragmática. El tratamiento se llevó a cabo durante dos semanas en la clínica en los tres grupos. Las modificaciones de comportamiento y de ejercicio de respiración diafragmática se continuaron en casa durante un mes.

Las manifestaciones clínicas, los parámetros de uroflujometría, y la orina residual postevaporizada fueron analizados antes y después de 6 semanas de terapia. Los resultados: Después del tratamiento, se observó una mejora significativa en la frecuencia de la defecación y la incontinencia fecal sólo en el grupo A $(\mathrm{P}<.001$ y $\mathrm{P}<.05$, respectivamente $)$. Estos niños demostraron una mejora significativa en los síntomas del tracto urinario inferior y en la orina residual postevaporizada $(\mathrm{P}<.001$ y $\mathrm{P}<.05$, respectivamente $)$. La curva de uroflujometría en forma de campana se observó en el 73,3\% de los pacientes del grupo $\mathrm{A}(\mathrm{P}<.001)$.

\section{Crioterapia en pacientes pediátricos}

En la literatura existen abundante información con respecto a los efectos fisiológicos de la crioterapia, siendo la disminución de la temperatura, el efecto principal a partir del cual, se generan otros cambios en diferentes tejidos y/o sistemas. Crioterapia se clasifica como una modalidad de termoterapia superficial que se basa en la aplicación del frío como agente terapéutico, la reducción de la temperatura del organismo tiene como finalidad aliviar el dolor, así como la reducción del edema, generando una respuesta tisular.
En el paciente pediátrico resulta contradictoria su aplicación, ya que se tiene la creencia cultural que la aplicación de esta, podría causarle enfermedades respiratorias al infante, revisaremos que dice la evidencia científica:

La Dra. Claudia Mendoza publica en la Revista Mexicana de Medicina Física y Rehabilitación en el año 2001, acerca de un programa de rehabilitación pre y postoperatorio para pacientes pediátricos con tumores óseos malignos primarios en extremidades, manejados con cirugía de salvamento, en este se aplica la crioterapia durante las primeras 24 horas con el objetivo de control de dolor y edema, se aplica en la extremidad intervenida, flexión de $40^{\circ}$, elevación en bloque, cambio de posición cada 2 horas. Aunque este trabajo no está enfocado a la crioterapia, es utilizada como beneficio para el paciente, al obtenerse como resultado que existen mejorías en la funcionalidad de la extremidad repercutiendo directamente sobre la vida del paciente. (Mendoza, et al, 2001).

Caroline en el 2019, confirma lo evidenciado en el artículo en su artículo: Gestión del dolor de niños y adolescentes durante el periodo post - trasplante de células tronco hematopoyéticas: una revisión integradora, utilizando la crioterapia por los enfermeros como estrategia para alivio de dolor (Graziele, et at 2019)

Algunos autores mencionan también a la Crioterapia para pacientes con parálisis cerebral infantil tipo espástica, como lo es Collado, en su estudio menciona utilizar la crioterapia al finalizar la sesión para ayudar a estimular termorreceptores que inhiben a las neuronas que desencadenan la espasticidad (Collado, 2018)

Bustamante en el 2018 plantea la Crioterapia como parte de un protocolo de tratamiento para la primera infancia de 3-5 minutos por 3 veces a la semana, se debe tener un control permanente de la temperatura, que sea adecuada y estable contando el tiempo exacto por parte del Fisioterapeuta. 


\section{Metodología}

Se realiza una revisión sistemática, entre el 06 de julio y el 30 de octubre del 2020. Las revisiones sistemáticas son investigaciones científicas en las que las unidades de análisis son los estudios originales primarios, a partir de los cuales se pretende contestar a una pregunta de investigación claramente formulada mediante un proceso sistemático, explícito y reproducible.

El proceso de elaboración de esta investigación, alcanzó 6 etapas: 1. Definición de la pregunta de investigación, 2. Selección de los criterios de inclusión y exclusión, 3. Localización y selección de los estudios relevantes, 4. Extracción de datos de los estudios primarios, 5. Análisis y presentación de los resultados, por último 6. Interpretación de los resultados.

En la primera etapa, se formuló la siguiente pregunta de investigación: ¿Los agentes físicos son benéficos para los pacientes pediátricos?

En la segunda etapa se deciden incluir todos los estudios que sean de tipo observacionales, descriptivos, cohortes y casos control, así como meta-análisis, en idiomas tanto español e inglés, se excluyeron artículos que reportaran pacientes de más de 18 años, y que no usaran agentes físicos, artículos de revisión que no involucren investigación son seres humanos y estudios realizados exclusivamente con pacientes adultos. Para los criterios de exclusión y de inclusión se uso la escala de PEDro, la puntuación inferior a 6 puntos, es para estudios controlados y aleatorios (Tabla 1)

\begin{tabular}{|c|c|c|c|}
\hline \multicolumn{4}{|c|}{ Escala de PEDro } \\
\hline \multicolumn{2}{|c|}{ Criterios } & Sí & No \\
\hline 1. & $\begin{array}{l}\text { Criterios de elegibilidad fueron } \\
\text { especificados (no se cuenta para } \\
\text { el total) }\end{array}$ & 1 & 0 \\
\hline 2. & $\begin{array}{l}\text { Sujetos fueron ubicados } \\
\text { aleatoriamente en grupos }\end{array}$ & 1 & 0 \\
\hline 3. & $\begin{array}{l}\text { La asignación a los grupos fue } \\
\text { encubierta }\end{array}$ & 1 & 0 \\
\hline 4. & $\begin{array}{l}\text { Los grupos tuvieron una línea de } \\
\text { base similar en los indicador es } \\
\text { de pronostico más importante }\end{array}$ & 1 & 0 \\
\hline 5. & $\begin{array}{l}\text { Hubo cegamiento para todos los } \\
\text { sujetos }\end{array}$ & 1 & 0 \\
\hline 6. & $\begin{array}{l}\text { Hubo cegamiento para todos los } \\
\text { terapeutas que administraron la } \\
\text { intervención }\end{array}$ & 1 & 0 \\
\hline
\end{tabular}

ECORFAN $^{\circledR}$ Todos los derechos reservados

\begin{tabular}{|l|l|r|r|}
\hline 7 & $\begin{array}{l}\text { Hubo cegamiento de todos los } \\
\text { asesores que midieron al menos } \\
\text { un resultado clave }\end{array}$ & 1 & 0 \\
\hline 8 & $\begin{array}{l}\text { Las mediciones de al menos un } \\
\text { resultado clave fueron obtenidas } \\
\text { en más del 85\% de los sujetos } \\
\text { inicialmente ubicados en los } \\
\text { grupos }\end{array}$ & 1 & 0 \\
\hline 9 & $\begin{array}{l}\text { Todos los sujetos medidos en los } \\
\text { resultados recibieron el } \\
\text { tratamiento o condición de } \\
\text { control tal como se les asignó, o } \\
\text { si no fue este el caso, los datos de } \\
\text { al menos uno de los resultados } \\
\text { clave fueron analizados con } \\
\text { intención de tratar }\end{array}$ & 1 & 0 \\
\hline 10 & $\begin{array}{l}\text { Los resultados de comparaciones } \\
\text { estadísticas entre grupos fueron } \\
\text { reportados en al menos un } \\
\text { resultado clave }\end{array}$ & 1 & \\
\hline 11 & $\begin{array}{l}\text { El estudio provee puntos y } \\
\text { mediciones de variabilidad para } \\
\text { al menos un resultado clave }\end{array}$ & 1 & \\
\hline
\end{tabular}

Tabla 1 Criterios de exclusión e inclusión usados en el estudio

Fuente: Elaboración Propia

La búsqueda se realizó en el 06 de julio y el 30 de octubre del 2020, en bases de información electrónica, como: Google Académico, Medigraphic, Fisiotherapy evidence Databases (PEDro), y Pubmed, utilizando los operadores booleanos "AND" para la combinación de los descriptores de: Ultrasonido terapéutico, Crioterapia, Hidroterapia, Agentes físicos, Electroterapia, pacientes pediátricos, niños, ultrasound and children, electrotherapy and pediatrics (Tabla 2).

\begin{tabular}{|c|c|c|}
\hline Descriptores: & Fuentes de datos: & $\begin{array}{c}\text { Total de } \\
\text { estudios } \\
\text { utilizados: }\end{array}$ \\
\hline $\begin{array}{l}\text { Ultrasonido } \\
\text { terapéutico }\end{array}$ & \multirow{5}{*}{ Google Académico } & 5 \\
\hline Uso de crioterapia & & 7 \\
\hline $\begin{array}{l}\text { Uso de la } \\
\text { hidroterapia }\end{array}$ & & 13 \\
\hline $\begin{array}{l}\text { Uso de los agentes } \\
\text { físicos terápeuticos }\end{array}$ & & 4 \\
\hline $\begin{array}{l}\text { Uso de la } \\
\text { electroterapia }\end{array}$ & & 5 \\
\hline $\begin{array}{l}\text { Ultrasonido } \\
\text { terapéutico }\end{array}$ & Medigraphic & 1 \\
\hline \multicolumn{3}{|l|}{$\begin{array}{l}\text { Niños y pacientes } \\
\text { pediátricos }\end{array}$} \\
\hline $\begin{array}{l}\text { Ultrasound and } \\
\text { children }\end{array}$ & $\begin{array}{l}\text { Physiotherapy } \\
\text { Evidence Database } \\
\text { (PEDro) }\end{array}$ & 3 \\
\hline $\begin{array}{l}\text { Electrotherapy and } \\
\text { pediatrics }\end{array}$ & Pubmed & 8 \\
\hline Total: 45 & & \\
\hline
\end{tabular}

Tabla 2 Descriptores usados en el estudio Fuente: Elaboración Propia

JAIME-ESTRADA, Sandra \& SASIA-ZAYAS, Karen. Revisión sistemática del uso de agentes físicos de pacientes pediátricos basado en la evidencia científica. Revista de Técnicas de la Enfermería y Salud. 2020 
Se realizó una selección de los artículos para ser parte de la muestra, tomando como referencia el cálculo del estadístico kappa, dicho estadístico, expresado de forma simple, mide el grado de acuerdo entre los revisores.

Por último, es importante tener en cuenta que todo el proceso de localización y selección de estudios se reportó correctamente en el diagrama de flujo los artículos identificados en cada fase, tomando en cuenta los eliminados y las causas de su eliminación (Figura 1)

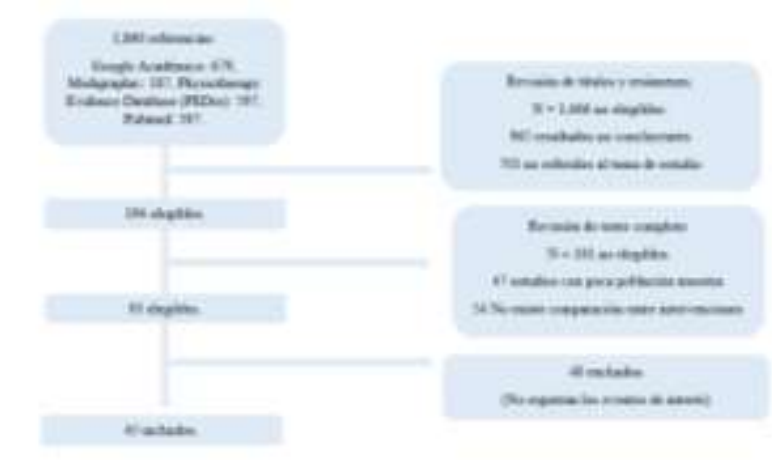

Figura 1 Diagrama de flujo para la selección de estudios Fuente: Elaboración propia

Para los estudios seleccionados, se hizo un registro en Microsoft Office Excel ${ }^{\circledR}$ 2016, fue diseñada por las autoras, tomando en cuenta: autor o autores, año de publicación, editorial o revista, nivel de evidencia y resultados Para el análisis de los resultados y la clasificación en cuanto al nivel de evidencia, fue realizado de acuerdo con la escala de Oxford, del Center for Evidence- Base Medicine, Oxford, que clasifica el nivel de evidencia en: grado de recomendación A, B y $\mathrm{C}, \mathrm{y}$ un nivel de evidencia 1 revisión sistemática, nivel 2: estudios de cohortes y ensayos clínicos aleatorios, nivel 3: revisión sistemática de casos y controles, serie de casos, y estudios de control individual. (Tabla 3)

\begin{tabular}{|c|c|c|}
\hline \multicolumn{3}{|c|}{ Escala de Oxford } \\
\hline $\begin{array}{l}\text { Grado de } \\
\text { recomendación }\end{array}$ & $\begin{array}{l}\text { Nivel de } \\
\text { evidencia }\end{array}$ & Fuente \\
\hline \multirow[t]{4}{*}{ A } & $1 \mathrm{a}$ & $\begin{array}{l}\text { Revisión sistemática de } \\
\text { ECA, con homogeneidad, } \\
\text { o sea que incluya estudios } \\
\text { con resultados } \\
\text { comparables y en la } \\
\text { misma dirección }\end{array}$ \\
\hline & $1 \mathrm{~b}$ & $\begin{array}{l}\text { ECA individual (con } \\
\text { intervalos de confianza } \\
\text { estrechos) }\end{array}$ \\
\hline & $1 \mathrm{c}$ & $\begin{array}{l}\text { Eficacia demostrada por la } \\
\text { práctica clínica y no por la } \\
\text { experimentación }\end{array}$ \\
\hline & $2 \mathrm{a}$ & $\begin{array}{l}\text { Revisión sistemática de } \\
\text { estudios de cohortes, con } \\
\text { homogeneidad, o sea que } \\
\text { incluya estudios con } \\
\text { resultados comparables y } \\
\text { en la misma dirección }\end{array}$ \\
\hline \multirow[t]{4}{*}{ B } & $2 \mathrm{~b}$ & \begin{tabular}{llll} 
Estudio de & \multicolumn{2}{r}{ cohortes } \\
individual y & \multicolumn{2}{r}{ ensayos } \\
clínicos aleatorios de & baja \\
calidad (< & $80 \%$ & de \\
seguimiento) & & \\
\end{tabular} \\
\hline & $2 \mathrm{c}$ & \begin{tabular}{|ll}
$\begin{array}{l}\text { Investigación } \\
\text { resultados en salud }\end{array}$ & de \\
\end{tabular} \\
\hline & $3 \mathrm{a}$ & $\begin{array}{l}\text { Revisión sistemática de } \\
\text { estudios de casos y } \\
\text { controles, } \\
\text { homogeneidad, o sea que } \\
\text { incluya estudios con } \\
\text { resultados comparables y } \\
\text { en la misma dirección }\end{array}$ \\
\hline & $3 \mathrm{~b}$ & \begin{tabular}{|l}
$\begin{array}{l}\text { Estudios de casos y } \\
\text { controles individuales }\end{array}$ \\
\end{tabular} \\
\hline $\mathrm{C}$ & 4 & $\begin{array}{l}\text { Serie de casos y estudios } \\
\text { de cohortes y casos y } \\
\text { controles de baja calidad }\end{array}$ \\
\hline
\end{tabular}

Tabla 3 Escala de Oxford para clasificar el nivel de evidencia

\section{Agradecimiento}

Agradecemos a la Universidad Tecnológica del Suroeste de Guanajuato por el apoyo para la publicación de esta investigación.

\section{Conclusiones}

De los estudios analizados se puede concluir que el uso de agentes físicos es beneficioso en los pacientes pediátricos.

Basado en la evidencia científica se puede obtener que existen pocas contraindicaciones en su aplicación con los niños. 
Una de las mayores contribuciones de este estudio es que esta investigación permite la búsqueda, la evaluación crítica y la síntesis de las evidencias disponibles sobre el tema investigado, siendo su producto final el estado actual del conocimiento del tema investigado, la implementación e intervenciones efectivas en la atención de la salud.

\section{Referencias}

F.J.Pavez Ulloa. (April 2009). Agentes físicos superficiales y dolor. Análisis de su eficacia a la luz de la evidencia científica. Revista de la Sociedad Española del Dolor, 16, 182-189.

Grillo PM, López PA. La Fisioterapia: sus orígenes y su actualidad. Acta Med Cent. 2016;10(3):88-90.

Calvo-Muñoz, A. Gómez-Conesa y J. SánchezMeca. (Junio 2012). Eficacia de los tratamientos de fisioterapia para el dolor lumbar en niños y adolescentes. Revisión sistemática. Revista de la Sociedad Española del Dolor, 19, 138-146.

PR Arriaga Hernández, M. Herrera Marmolejo, A.R. Parodi Carbajal. (Diciembre 2010). Efectividad de la aplicación de ultrasonido terapéutico y ejercicio de estiramiento a músculos isquiotibiales en niños con parálisis cerebral tipo diparesia espástica. Rehabilitación, 44 (4), 326-330.

I.I.Cigarroa, F.A.Palomera, J.P.Pino, H.C.Hernández, S.A.Sepúlveda, C.A.Soto, R.E.Zapata-Lamana. (Junio 2016). Ganancia de extensión de rodilla usando ultrasonido terapéutico y estiramiento pasivo en pacientes con parálisis cerebral espástica leve. Fisioterapia, 38, 118-126.

Marcos Almirón. (Agosto 2019). Breve reseña sobre el ultrasonido terapéutico. MEDICINA CLÍNICA Y SOCIAL, 3.

Villaseñor Moreno, Julio César; Escobar Reyes, Victor Hugo; de la Lanza Andrade, Laura Patricia; Guizar Ramírez, Brenda Irma. ( abriljunio 2013). Síndrome de dolor miofascial. Epidemiologia, fisiopatología, diagnóstico y tratamiento Revista de Especialidades MédicoQuirúrgicas. Revista de Especialidades MédicoQuirúrgicas, 2, 148-157.
Flores Toapanta, D. L. (2014-0604T15:36:26Z). Eficacia de la aplicación Hidroterapéutico Hallwick en niños con parálisis cerebral infantil en las instalaciones del Complejo Acuático de la Universidad Técnica del Norte 2013 (Bachelor's thesis). Recuperado http://repositorio.utn.edu.ec/handle/123456789/ 3612

Hernández DPA, Díaz TD, Sánchez MK, et al. Tortícolis muscular congénita asociada a asimetría facial y malformación de Chiari I. Rev Cub de Med Fis y Rehab. 2019;11(2):110.

Marcelino Prudencio Mamani Pacari; Dr. Yercin Mamani Ortiz, Dr. Daniel Illanes Velarde; Lic. Marcos Rene Rocha Albino. (2016). Radiofrecuencia vs Corriente Farádica en la rehabilitación funcional de pacientes con parálisis facial periférica. Revista Científica Ciencia Médica, 19, 5-13.

Khamis S, Herman T, Krimus S, Danino B. Is functional electrical stimulation an alternative for orthotics in patients with cerebral palsy? A literature review. Eur J Paediatr Neurol. 2018 Jan;22(1):7-16.

doi: 10.1016/j.ejpn.2017.10.004. Epub 2017 Oct 14. PMID: 29102346.

Elnaggar RK, Elbanna MF. Evaluation of independent versus integrated effects of reciprocal electrical stimulation and botulinum toxin-A on dynamic limits of postural stability and ankle kinematics in spastic diplegia: a single-blinded randomized trial. Eur J Phys Rehabil Med. 2019 Apr;55(2):241-249. doi: 10.23736/S1973-9087.18.05196-1. Epub 2018 Jun 14. PMID: 29904047.

Wright AJ, Haddad M. Electroneurostimulation for the management of bladder bowel dysfunction in childhood. Eur J Paediatr Neurol. 2017 Jan;21(1):67-74. doi: 10.1016/j.ejpn.2016.05.012. Epub 2016 May 27. PMID: 27328864.

Tubaro A, Puccini F, De Nunzio C. The management of overactive bladder: percutaneous tibial nerve stimulation, sacral nerve stimulation, or botulinum toxin? Curr Opin Urol. 2015 Jul;25(4):305-10. doi: 10.1097/MOU.0000000000000180. PMID: 26049873.

JAIME-ESTRADA, Sandra \& SASIA-ZAYAS, Karen. Revisión sistemática del uso de agentes físicos de pacientes pediátricos basado en la evidencia científica. Revista de Técnicas de la Enfermería y Salud. 2020 
Sousa, Graziele Caroline Cardoso de, Mercês, Nen Nalú Alves das, Silva, Lara Adrianne Garcia Paiano da, \& Macedo, Alini. (2019). Gestión del dolor de niños y adolescentes durante el período post- trasplante de célulastronco hematopoyéticas: una revisión integradora. Enfermería Global, 18(53), 535581. Epub 14 de octubre de 2019.https://dx.doi.org/10.6018/eglobal.18.1.30 2991

Moll I, Vles JSH, Soudant DLHM, Witlox AMA, Staal HM, Speth LAWM, JanssenPotten YJM, Coenen M, Koudijs SM, Vermeulen RJ. Functional electrical stimulation of the ankle dorsiflexors during walking in spastic cerebral palsy: a systematic review. Dev Med Child Neurol. 2017 Dec;59(12):12301236. doi: 10.1111/dmcn.13501. Epub 2017 Aug 17. PMID: 28815571.

Dombek K, Costa Monteiro LM, Fontes JM, Ramos EG. Immediate effect of transcutaneous electrical nerve stimulation on urodynamic parameters of children with myelomeningocele. Neurourol Urodyn. 2019 Nov;38(8):2351-2358. doi: 10.1002/nau.24155. Epub 2019 Sep 4. PMID: 31486143.

Rego RMP, Machado NC, Carvalho MA, Graffunder JS, Ortolan EVP, Lourenção PLTA. Transcutaneous posterior tibial nerve stimulation in children and adolescents with functional constipation: A protocol for an interventional study. Medicine (Baltimore). 2019 Nov;98(45):e17755. doi: 10.1097/MD.0000000000017755. PMID: 31702626 ; PMCID: PMC6855522.

Dra. Claudia Mendoza Martínez,* Dra. Betty Coutiño León,* Dr. Alejandro Medina Salas,* Dr. Ignacio Mora Magaña. (2001). Programa de rehabilitación pre y posoperatorio para pacientes pediátricos con tumores óseos malignos primarios en extremidades, manejados con cirugía de salvamento. Revista Mexicana de Medicina Física y Rehabilitación, 13, 44-49.

Zivkovic VD, Stankovic I, Dimitrijevic L, Kocic M, Colovic H, Vlajkovic M, Slavkovic A, Lazovic M. Are Interferential Electrical Stimulation and Diaphragmatic Breathing Exercises Beneficial in Children With Bladder and Bowel Dysfunction? Urology. 2017 Apr;102:207-212. doi: 10.1016/j.urology.2016.12.038. Epub 2016 Dec 28. PMID: 28040503.

ISSN: 2523-0352

ECORFAN ${ }^{\circledR}$ Todos los derechos reservados
Mamani Pacari, Marcelino Prudencio, Mamani Ortiz, Yercin, Illanes Velarde, Daniel, \& Rocha Albino, Marcos Rene. (2016). Radiofrecuencia vs Corriente Farádica en la rehabilitación funcional de pacientes con parálisis facial periférica. Revista Científica Ciencia Médica, 19(2), 5-13. Recuperado en 08 de diciembre de 2020, de http://www.scielo.org.bo/scielo.php?script=sci_ arttext\&pid=S1817$74332016000200002 \& \operatorname{lng}=$ es\&tlng=es.

Moro-De Faes G, Serrano-Moyano B, CantarinExtremera V, Moreno-Vinues B, GarciaFernandez M, Perez-Jimenez MA, RiveroMartin MB, Garcia-Ezquiaga J, DuatRodriguez A, Ruiz-Falco Rojas ML. Diez años de experiencia con el estimulador del nervio vago en una poblacion pediatrica [Ten years' experience with vagus nerve stimulation in a paediatric population]. Rev Neurol. 2018 Nov 16;67(10):382-386. Spanish. PMID: 30403281.

Fuentes-Pita P, Gomez-Lado C, Dacruz D, Eiris-Punal J, Prieto-Gonzalez A, Castro-Gago M. Analisis retrospectivo sobre el efecto del estimulador vagal implantado en pacientes pediatricos con epilepsia refractaria [Retrospective analysis of the effect of a vagus nerve stimulator implanted in paediatric patients with refractory epilepsy]. Rev Neurol. 2016 Jul 1;63(1):11-8. Spanish. PMID: 27345275 .

Flesler S, Reyes G, Fortini S, Ramos B, Cersosimo R, Bartuluchi M, Caraballo R. Estimulador del nervio vago: tratamiento en 158 pacientes pediatricos con un largo seguimiento [Vagus nerve stimulation: treatment of 158 pediatric patients with a longterm follow-up]. Rev Neurol. 2017 Jun 1;64(11):496-501. Spanish. PMID: 28555455.

Pool D, Elliott C, Bear N, Donnelly CJ, Davis C, Stannage K, Valentine J. Neuromuscular electrical stimulation-assisted gait increases muscle strength and volume in children with unilateral spastic cerebral palsy. Dev Med Child Neurol. 2016 May;58(5):492-501. doi: 10.1111/dmcn.12955. Epub 2015 Nov 11. PMID: 26555148. 
Ladi-Seyedian SS, Sharifi-Rad L, Kajbafzadeh AM. Pelvic floor electrical stimulation and muscles training: a combined rehabilitative approach for management of non-neuropathic urinary incontinence in children. J Pediatr Surg. 2019 Apr;54(4):825-830. doi: 10.1016/j.jpedsurg.2018.06.007. Epub 2018 Jun 11. PMID: 29960741.

Andreoli SM, Wilson BL, Swanson C. Neuromuscular electrical stimulation improves feeding and aspiration status in medically complex children undergoing feeding therapy. Int J Pediatr Otorhinolaryngol. 2019 Dec;127:109646. doi: 10.1016/j.ijporl.2019.109646. Epub 2019 Aug 19. PMID: 31450168.

Bosques G, Martin R, McGee L, Sadowsky C. Does therapeutic electrical stimulation improve function in children with disabilities? A comprehensive literature review. J Pediatr Rehabil Med. 2016 May 31;9(2):83-99. doi: 10.3233/PRM-160375. PMID: 27285801.

Claudia Yaneth Franco Monsalve, Zorika María Guerra Corena, María Patricia Otero Samudio. (Septiembre-diciembre de 2007). Estudio de caso: terapia manual en una paciente de 18 años con escoliosis juvenil idiopática. Rev. Cienc. Salud. Bogotá (Colombia), 5, 78-90.

Unger M, Jelsma J, Stark C. Effect of a trunktargeted intervention using vibration on posture and gait in children with spastic type cerebral palsy: a randomized control trial. Dev Neurorehabil. 2013;16(2):79-88. doi: 10.3109/17518423.2012.715313. PMID: 23477461 .

Ilkay Karabay, MD Go“ khan Tuna O” ztu“ rk, MD Fevziye U" nsal Malas, MD Murat Kara, MD Tu“ lay Tiftik, MD Murat Erso“ z, MD Levent O" zc sakar, MD. (September 2015). Short-Term Effects of Neuromuscular Electrical Stimulation on Muscle Architecture of the Tibialis Anterior and Gastrocnemius in Children with Cerebral Palsy Preliminary Results of a Prospective Controlled Study. 6Octubre-2020, de American Journal of Physical Medicine \& Rehabilitation Copyright Sitio web:

https://fac.ksu.edu.sa/sites/default/files/17nmesanterior_tiable_muscles.pdf
Michelle H. Cameron. (2013). Agentes físicos en rehabilitación De la investigación a la práctica. España: Elsevier.

Porro NJ, Estévez PA, Rodriguez GA, et al. Guía para la rehabilitación de la fibromialgia. Rev Cub de Reu. 2015;17(Suppl: 1):147-156.

González MM, Rodríguez RME. Protocolo de rehabilitación en el paciente con tortícolis muscular congénita. Rev Cub de Med Fis y Rehab. 2013;5(2):153-165.

Shi X, Yu W, Wang T, Shu Q, Wang C, Yang $\mathrm{X}$, Liu C, Guo C. A comparison of the effects of electroacupuncture vs transcutaneous electrical nerve stimulation for pain control in knee osteoarthritis: A protocol for network meta-analysis of randomized controlled trials. Medicine (Baltimore). 2019 Jul;98(28):e16265. doi: 10.1097/MD.000000000016265. PMID: 31305408; PMCID: PMC6641830.

Armstrong EL, Boyd RN, Kentish MJ, Carty $\mathrm{CP}$, Horan SA. Effects of a training programme of functional electrical stimulation (FES) powered cycling, recreational cycling and goaldirected exercise training on children with cerebral palsy: a randomised controlled trial protocol. BMJ Open. 2019 Jun 17;9(6):e024881. doi: 10.1136/bmjopen-2018024881. PMID: 31213443; PMCID: PMC6589006.

Wang H, Song GF, Nie J, Xu XH, Zhang Y, Liu JR. Electrical stimulation for limb spasticity in children with traumatic brain injury: Study protocol for a systematic review of randomized controlled trial. Medicine (Baltimore). 2019 Feb;98(8):e14515. doi: 10.1097/MD.0000000000014515. PMID: 30813153; PMCID: PMC6408011.

Laufer Y, Shtraker H, Elboim Gabyzon M. The effects of exercise and neuromuscular electrical stimulation in subjects with knee osteoarthritis: a 3-month follow-up study. Clin Interv Aging. 2014 Jul 17;9:1153-61. doi: 10.2147/CIA.S64104. PMID: 25083133; PMCID: PMC4108455. 
Magalhães P, Figueirêdo BB, Vasconcelos A, de Andrade ÉM, Dornelas de Andrade A, Reinaux C. Is transcutaneous electrical muscle stimulation an alternative for preventing acquired muscle weakness in the pediatric intensive care unit? A scoping review. Pediatr Pulmonol. 2019 Aug;54(8):1108-1116. doi: 10.1002/ppul.24293. Epub 2019 May 27. PMID: 31134767.

Berggren J, Baker LL. Therapeutic application of electrical stimulation and constraint induced movement therapy in perinatal brachial plexus injury: A case report. J Hand Ther. 2015 AprJun;28(2):217-20; quiz 221. doi: 10.1016/j.jht.2014.12.006. Epub 2014 Dec 17. PMID: 25841560.

Razumov AN, Pogonchenkova IV, Khan MA, Lyan NA, Vakhova EL, Mikitchenko NA. Primenenie impul'snogo nizkochastotnogo élektrostaticheskogo polia $\mathrm{v}$ pediatrii [The application of the pulsed low-frequency electrostatic field in pediatrics]. Vopr Kurortol Fizioter Lech Fiz Kult. 2019;96(1):55-62. Russian. doi: 10.17116/kurort20199601155. PMID: 30724883.

Epperson HE, Sandage MJ. Neuromuscular Development in Neonates and Postnatal Infants: Implications for Neuromuscular Electrical Stimulation Therapy for Dysphagia. J Speech Lang Hear Res. 2019 Aug 15;62(8):2575-2583. doi: 10.1044/2019_JSLHR-S-18-0502. Epub 2019 Jul 24. PMID: 31343903.

Jørgensen CS, Kamperis K, Borch L, Borg B, Rittig S. Transcutaneous Electrical Nerve Stimulation in Children with Monosymptomatic Nocturnal Enuresis: A Randomized, DoubleBlind, Placebo Controlled Study. J Urol. 2017 Sep;198(3):687-693.

doi: 10.1016/j.juro.2017.04.082. Epub 2017 Jul 23. PMID: 28747281. 